\title{
Transport pilot tests on thickened tailings to determine the effect of pumping distance on tailings sedimentation
}

\author{
P. Chacón ARCADIS, Chile \\ S. Barrera ARCADIS, Chile
}

\section{Abstract}

The extreme aridness of northern Chile makes tailings processes in the region have, as one of their main objectives, the recovery of most of the water used in the concentration process before the slurry is discharged in the tailings impoundment. A way of reaching this objective is to increase the degree of thickening using current technology. However, in such a scenario, the transport of tailings with high solids content is a limiting factor in the hydraulic design of the global management system since it is a challenge to ensure that the slurry does not settle out in transport systems longer than $2 \mathrm{~km}$. This is why it is imperative to study the behaviour of the transport systems of highly thickened tailings.

In the Cerro Negro Norte Project, a technical-economic analysis was prepared for several options of tailings thickening and transport between the plant and the impoundment (Barrera and Hills, 2009) using centrifugal pumps which considered the execution of pilot tests in a pumping loop. The first stage of the tests had the objective of validating the theoretical hydraulic model used in the design of the thickened tailings pumping and determining the maximum level of thickening that may be pumped with centrifugal pumps. The second stage consisted in operating the pumping loop for a longer period to detect the effect of the transport distance in the sedimentation of the tailings.

The purpose of this paper is to: (i) present the main results of the long term pilot tests done with thickened tailings in conditions of laminar flow; (ii) compare the recorded results with the theoretical models; (iii) analyse and interpret the main variations in time detected in the behaviour of the pumping loop; and (iv) provide comments and design recommendations for these types of projects.

\section{$1 \quad$ Introduction and objective}

The technology of paste and thickened tailings has become one of the fundamental pillars of the process of reducing the consumption of water by concentrating the tailings before being deposited in an impoundment. As a consequence, tailings transport systems are required to transport tailings with higher solids content. This has resulted in the need to investigate hydraulic design aspects where knowledge is limited as well as in the application of new theoretical models and the performance of tests at a larger scale than laboratory scale, commonly called 'pilot tests'.

The main objective of this study is to establish the type of flow as a function of the velocity (laminar, transition or turbulent) and the marked limits between them. With this knowledge it is possible to determine in which conditions the slurry will not sediment in short and long transports distances, thereby preventing that the hydraulic tailings transport becomes a limit to the maximum thickening level possible by the system and, therefore, the reduction in the consumption of fresh water.

Although there are no standard tests for any type of tailings - much less for the study of thickened tailings transport - the pilot tests have become a practical tool and ever more common for the support of decisions in engineering projects. In the Cerro Negro Norte Project, a technical-economic analysis was prepared for several options of tailings thickening and transport between the plant and the impoundment (Barrera and 
Hills, 2009) which considered the performance of pilot tests in a pumping loop with the objective of (i) estimating the maximum level of thickening that may be pumped with centrifugal pumps, and (ii) detect the effect of distance on tailings sedimentation in the transport (operation of the pumping loop for a longer period of time).

This paper has the objectives of: (i) presenting the main results of the prolonged period pilot tests done with thickened tailings in laminar flow conditions; (ii) comparing the real with the theoretical results; (iii) interpreting and analysing the significant time variations detected in the behaviour of the pumping loop; and (iv) providing design comments and recommendations for this type of projects.

\section{Theoretical model}

The tests have the objective of validating the theoretical model used for the design of thickened tailings pumping, determining the maximum level of thickening that may be pumped with centrifugal pumps, and making an estimate of the sedimentation in the pipeline under pressure.

For these effects, we compared the effective measurements of head loss and friction factors in the pumping loop with the expected theoretical values. In this manner, an adequate adjustment between the theoretical and the measured data implies the validation of the theoretical model used.

In this case, the tailings slurry has been modelled as non-Newtonian fluid and its head loss is calculated according to the expression of Darcy:

$$
J=f\left(\frac{V^{2}}{2 g D_{i}}\right)
$$

where:

$$
\begin{array}{lll}
\mathrm{J} & = & \text { head loss }(\mathrm{m} / \mathrm{m}) . \\
\mathrm{f} & = & \text { friction coefficient. } \\
\mathrm{V} & = & \text { average velocity of the slurry }(\mathrm{m} / \mathrm{s}) . \\
\mathrm{g} & = & \text { gravitational constant }\left(\mathrm{m} / \mathrm{s}^{2}\right) . \\
\mathrm{D}_{\mathrm{i}} & = & \text { internal diameter of the pipe }(\mathrm{m}) .
\end{array}
$$

For the case of turbulent fluid, the friction coefficient is calculated according to the expressions of Wilson and Thomas (1985) and Thomas and Wilson (1987).

In case of a laminar regime, the transport is sized with the equation of Haldenwang (2003), using equivalent hydraulic radio for pipe flow:

$$
\begin{gathered}
f=\frac{64}{\operatorname{Re}_{2}} \\
\operatorname{Re}_{2}=\frac{8 \rho V^{2}}{\tau_{y}+\frac{8 V \mu_{B}}{4 R_{H}}}
\end{gathered}
$$

where:

$R_{H} \quad=\quad$ equivalent hydraulic radius for pipe flow $=4 \cdot D_{i}(m)$.

$\rho=\quad$ density of the fluid $\left(\mathrm{kg} / \mathrm{m}^{3}\right)$.

$\tau_{\mathrm{y}}=$ yield stress of the fluid $(\mathrm{Pa})$. 
$\mu_{\mathrm{B}}$ $=\quad$ viscosity of Bingham (Pa.s).

The physical sense of the Re parameter is to represent inertial over viscous forces, therefore, it represents the turbulence of the flow, and its value increases. We have a flow with greater turbulence considering that a flow with well developed turbulence has $\mathrm{Re}_{2}$ in the range above 8,000-10,000. On the contrary, values of $\mathrm{Re}_{2}<4,000$ represent laminar flows. Obviously, in the in between range, we have flow in laminar-turbulent transition.

To estimate the settling limit velocity $\left(V_{L}\right)$, we have used the expression from Durand, modified by Rayo (1993):

$$
V_{L}=1.25 \cdot F_{L} \cdot \sqrt{2 \cdot g \cdot D_{i} \cdot\left(G_{S}-1\right)}
$$

where:

$V_{\mathrm{L}} \quad=\quad$ settling limit velocity $(\mathrm{m} / \mathrm{s})$.

$\mathrm{F}_{\mathrm{L}}=\quad$ parameter in function of the size and concentration of solids (McElvain and Cave, 1972).

Gs $\quad=\quad$ relative density of the solid with respect to the transporting fluid (a-dimensional).

\section{$3 \quad$ Pilot plant-pumping loop}

For the transport tests, we designed a pumping loop shown in Figure 1 that has two slurry preparation tanks of $5 \mathrm{~m}^{3}$ each. One tank uses a peristaltic pump to feed a $10 \mathrm{~m}^{3}$ tank. A centrifugal pump of $150 \mathrm{~kW}$ (with mechanical seal) pumps the slurry to the $1.5 \mathrm{~m}^{3}$ head tank of the system via a high density polyethylene pipe (HDPE) pipe of approximately $50 \mathrm{~m}$ located horizontally on the ground. The two easily interchangeable pipes used for the tests are HDPE PE100 PN8 with internal diameters of $200 \mathrm{~mm}$ and $160 \mathrm{~mm}$. The head tank discharges to an HDPE pipeline of $200 \mathrm{~mm}$ of approximately $50 \mathrm{~m}$ in length, where the tailings flow by gravity. This pipe is perforated in its upper part with the objective of facilitating the elimination of heat generated by the flow of slurry and limiting the temperature rise.

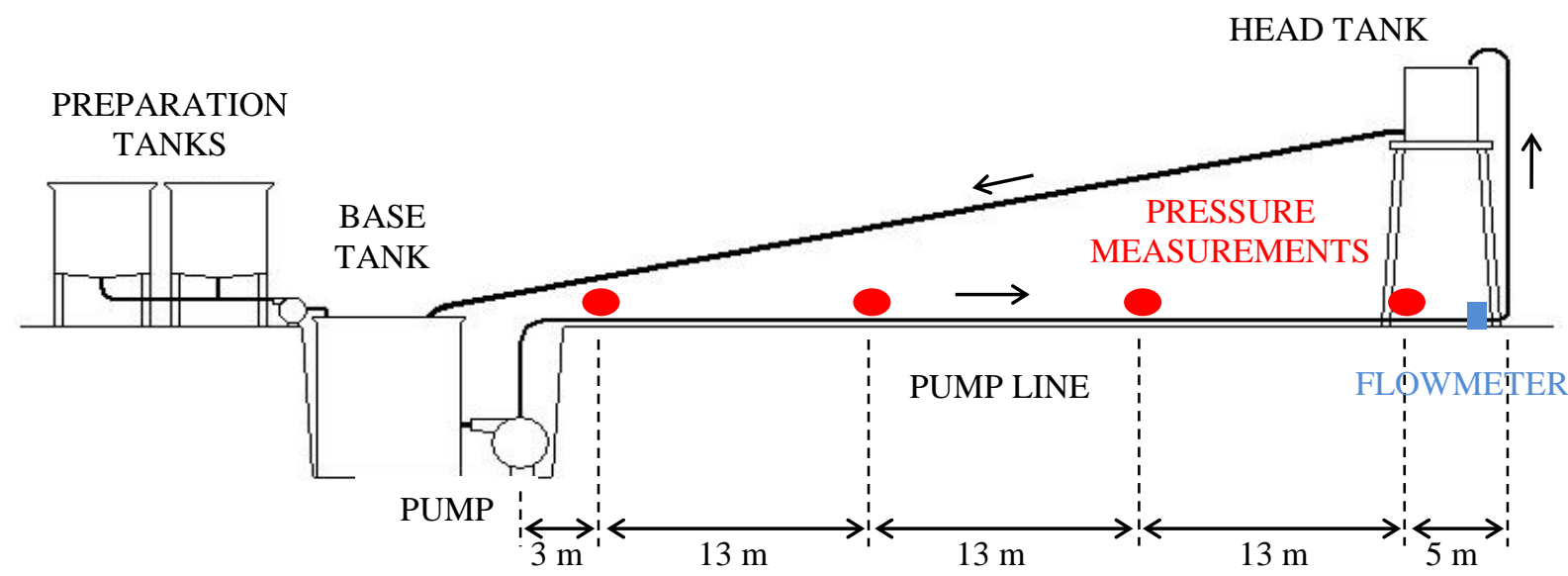

Figure 1 Sketch-facilities for the pump loop tests

Figure 2 presents a view of the facilities identifying some of the elements cited previously. 


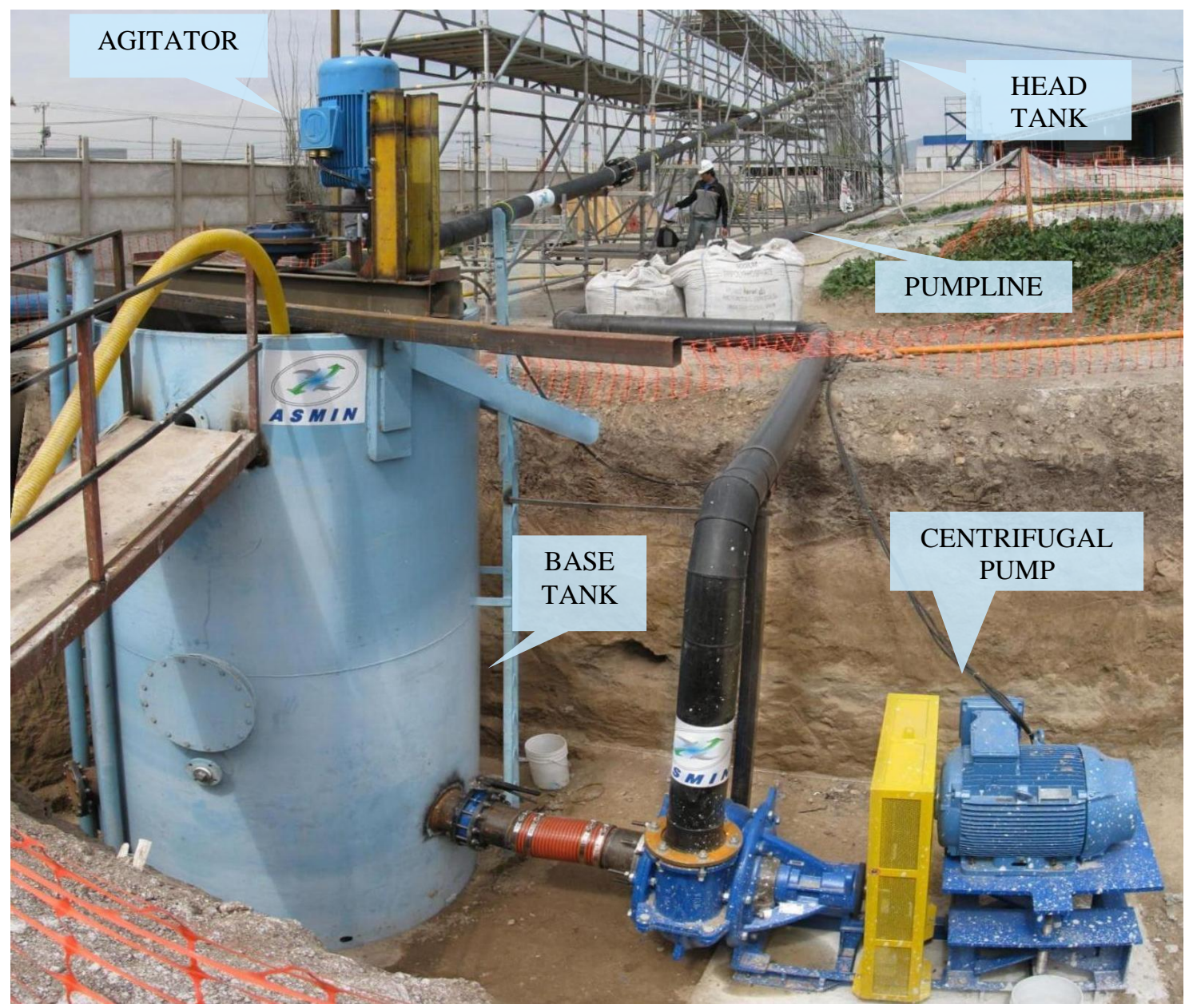

Figure 2 Facilities for the pump loop tests

\section{Test procedure}

To test this theory, input parameters needed for the theoretical model were measured before each test. These included:

- the dimensions of the pipe

- concentration by weight

- specific gravity

- grain size

- Bingham flow parameters using 'bob and cup' rheometer RS6000.

During the test, the flow rate and pressures in different locations throughout the pipeline were measured. This is used to estimate head loss and friction factors.

The test is done in two stages. The first stage has a duration of approximately 5-10 minutes and the objective is to measure flow, pressure and take samples for laboratory tests. Subsequent work with the laboratory results allows us to estimate the head loss and theoretical friction factor of each data set for comparison with the measured data.

The second stage of tests was done in view of the results of the tests described above. These consisted in operating the pump loop for several hours and recording every 10-15 minutes the flow and pressure in the pressure measuring devices located in the pipe. This was done to analyse if the behaviour of the recorded pressures and, therefore, the head loss in the pipe was stable with time or if there was some temporal 
variation that could be a reflection of an eventual gradual obstruction of the pipe by the presence of tailings sedimentation inside the pipe. Moreover, the grain size was measured after completion of every test.

These long duration tests were performed for concentrations in weight close to $\mathrm{Cw} 67-68 \%$ with the objective of trying to determine if at these concentrations there was evidence of sedimentation of the material. To test this, flow rates were selected so that the flow velocity within the pipe was slightly above $3 \mathrm{~m} / \mathrm{s}$, which is a typical design range for this type of tailings.

\section{$5 \quad$ Results}

The slurry was prepared at different solids content in weight for each test. For each case, a sample of the tailings that was sent to the laboratory was taken to measure the parameters in Table 1.

Table 1 Tailings parameters pump loop

\begin{tabular}{ccccccc}
\hline Test & $\begin{array}{c}\text { Concentration } \\
\text { Cw\% }\end{array}$ & $\mathbf{G}_{\mathbf{s}}$ & $\begin{array}{c}\tau_{\mathrm{y}} \\
(\mathbf{P a})\end{array}$ & $\begin{array}{c}\mu_{\mathrm{B}} \\
(\mathbf{P a} \cdot \mathbf{s})\end{array}$ & $\begin{array}{c}\text { Pipe } \\
\text { Diameter } \\
(\mathbf{m m})\end{array}$ & Flocculant \\
\hline 1 & 68.26 & 3.34 & 54.8 & 0.0581 & 160 & No \\
2 & 69.63 & 3.34 & 83.1 & 0.0811 & 200 & No \\
3 & 70.88 & 3.33 & 78.8 & 0.0893 & 200 & No \\
4 & 71.91 & 3.4 & 129.6 & 0.0929 & 160 & No \\
5 & 61.85 & 3.42 & 8 & 0.0198 & 160 & No \\
6 & 64.6 & 3.2 & 52.2 & 0.039 & 160 & No \\
$7\left(^{*}\right)$ & 67.7 & 3.22 & 107.4 & 0.0754 & 160 & No \\
$8\left(^{*}\right)$ & 68.5 & 3.2 & 130.7 & 0.0822 & 160 & No \\
\hline
\end{tabular}

(*) long duration tests.

Note: iron tailings with D50: $38 \mu \mathrm{m}$

Each sample was tested in the pump loop for different flows as shown in the following table.

Table 2 Flows recorded for each pump loop test

\begin{tabular}{clllllllllll}
\hline Test & & & \multicolumn{1}{c}{ Flows $\left[\mathrm{m}^{3} / \mathrm{s}\right]$} \\
\hline 1 & 0.067 & 0.064 & 0.06 & 0.049 & & & & & & \\
2 & 0.055 & 0.023 & 0.049 & 0.062 & & & & & & \\
3 & 0.08 & 0.068 & 0.062 & 0.053 & & & & & & \\
4 & 0.074 & 0.071 & 0.068 & 0.064 & 0.051 & 0.025 & 0.068 & & & & \\
5 & 0.14 & 0.133 & 0.138 & 0.132 & 0.127 & 0.133 & 0.122 & 0.111 & 0.08 & 0.078 & 0.064 \\
6 & 0.081 & 0.062 & 0.061 & 0.052 & 0.041 & & & & & & \\
$7(*)$ & $\sim 0.05$ & & & & & & & & & & \\
$8(*)$ & $\sim 0.05$ & & & & & & & & & & \\
\hline
\end{tabular}

$(*)$ long duration tests.

Following are the recorded values of the first stage plotted in function of the recorded head losses and flow velocities. 


\section{Head Loss measured (mcw) v/s Velocity $[\mathrm{m} / \mathrm{s}]$}

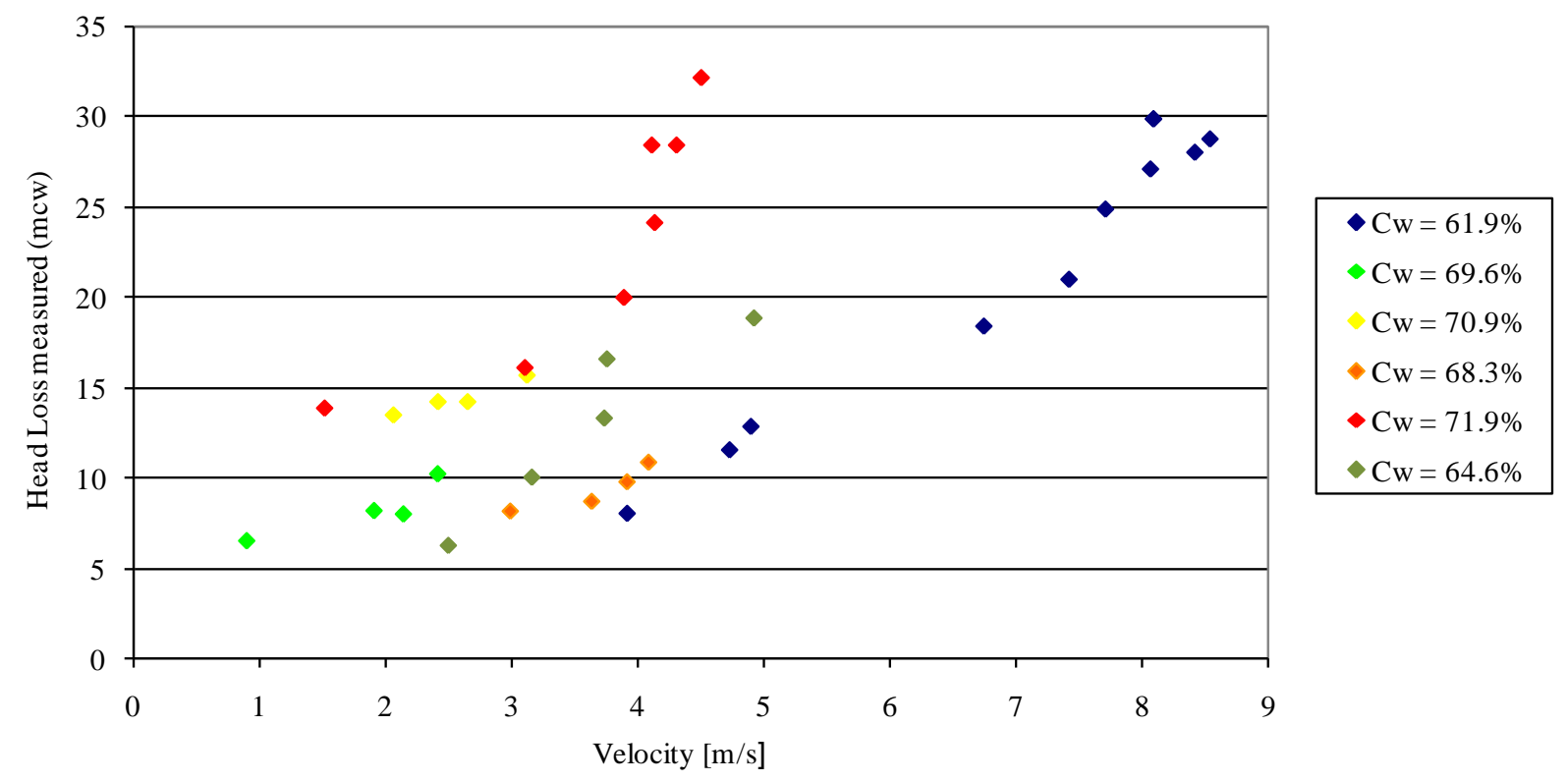

Figure 3 Variation of the recorded head losses ( $\mathrm{J} \%$ ) in function of the velocity $(\mathrm{V}[\mathrm{m} / \mathrm{s}])$

Figure 3 shows typical tendencies, that is, at greater flow velocities there are greater head losses. However, an additional analysis with regards to friction factors, allows us to better interpret the results of the tests. In Figure 4, we present these results together with the theoretical values in function of the nondimensional Reynolds Number, $\mathrm{Re}_{2}$.

We observe in Figure 4 that the measurements at $\mathrm{Cw}=61.9 \%$, located towards the right of the figure and that have a higher turbulent level adequately adjusts to the theoretical model of Wilson and Thomas (1985), which is valid for turbulent flows, that is, in the more horizontal part of the curve. On the other hand, the points located more to the left show a wide scatter, therefore the Haldenwang (2003) theoretical model for laminar flows represented by the descending line does not fit correctly. However, these data show an expected typical tailings behaviour with a descending line in the laminar flows, a horizontal line in turbulent flows and an intermediate transition zone.

The fitting of the theoretical model for turbulent flows during the duration of the tests and the greater dispersion of the points in the laminar-turbulent transition area does not allow us to assure the occurrence of sedimentation inside the pipe. Neither does the lack of evidence of sedimentation in some tests guarantee that this could not eventually occur in transport over greater lengths for which we need measurements in tests of prolonged durations. With this objective, we performed a second stage of additional long duration ( $\sim \mathrm{hr}$ ) in the pump loop as a way of verifying or supporting this aspect. 
Friction factor (f) v/s $\mathbf{R e}_{2}$

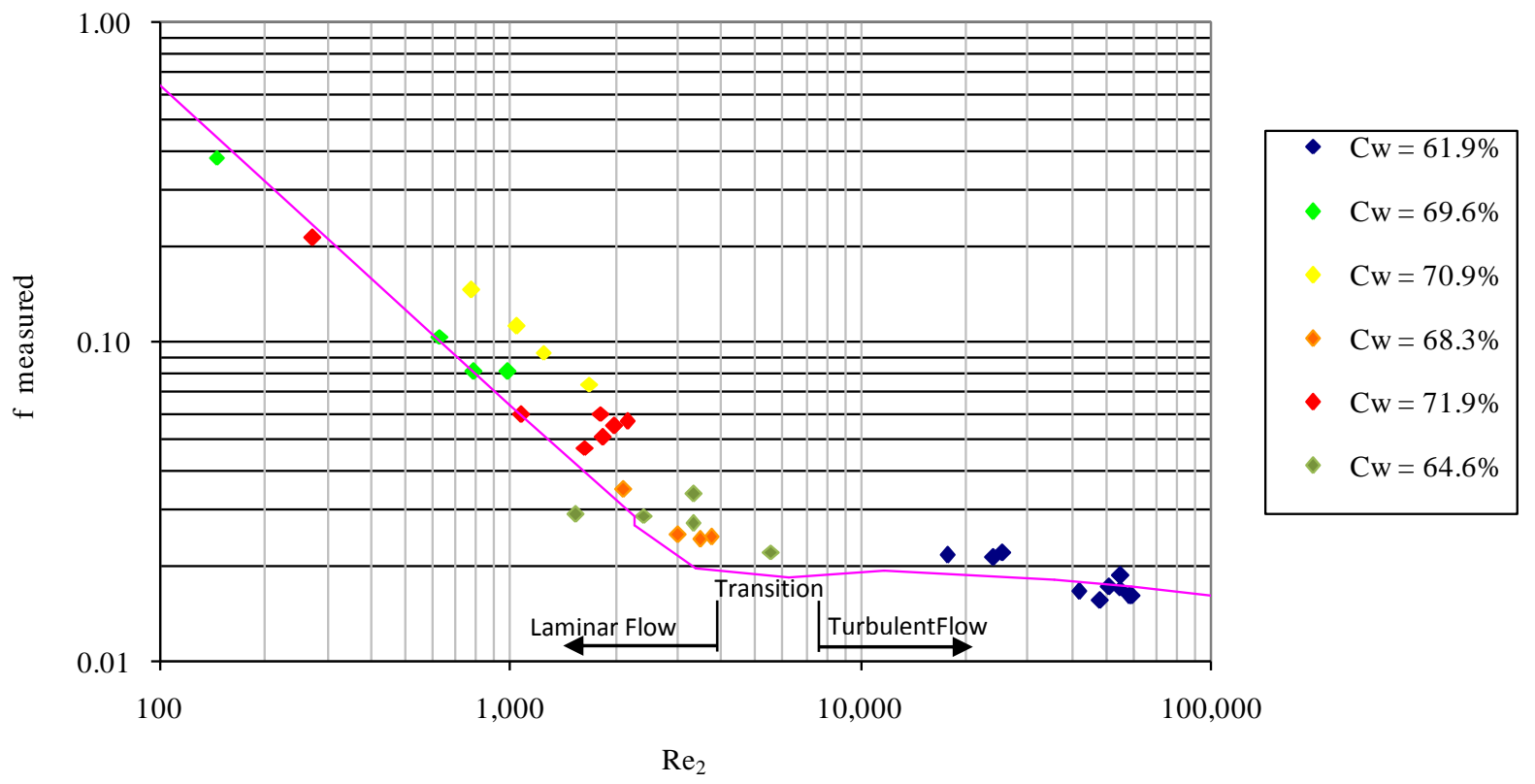

Figure 4 Comparison of friction factors (theoretical and recorded) in function of $\operatorname{Re}_{2}$

In the following figures, we present the results of the variation of the friction factor and the flow during the execution of Test No. 7.

\section{Friction factor v/s Time}

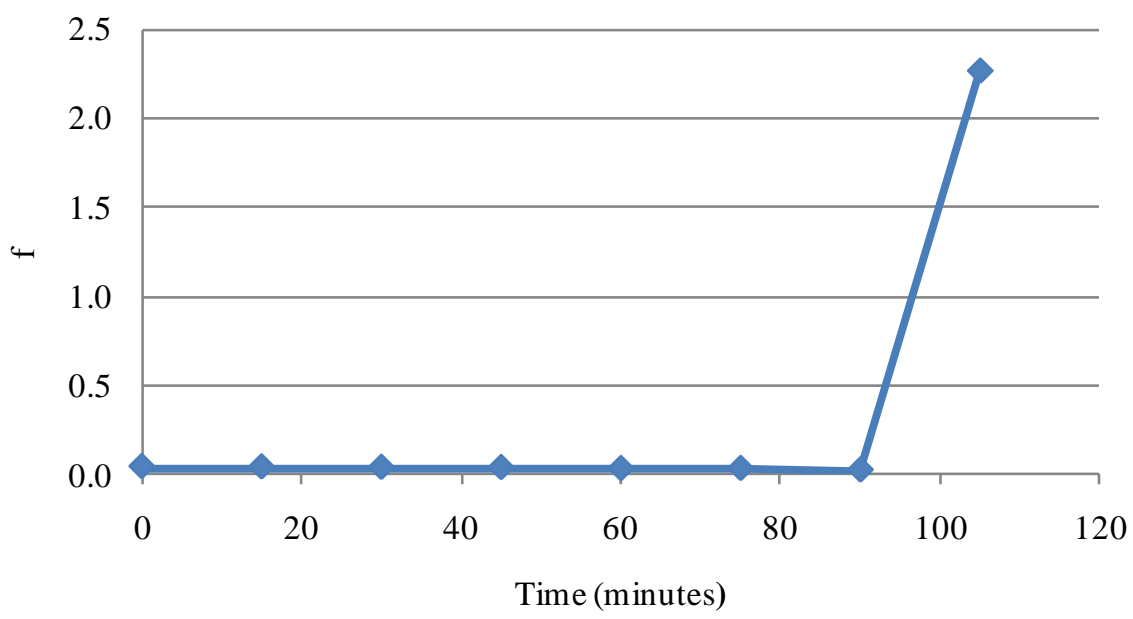

Figure 5 Variation of the friction factor measured in function of time (Test No. 7) 


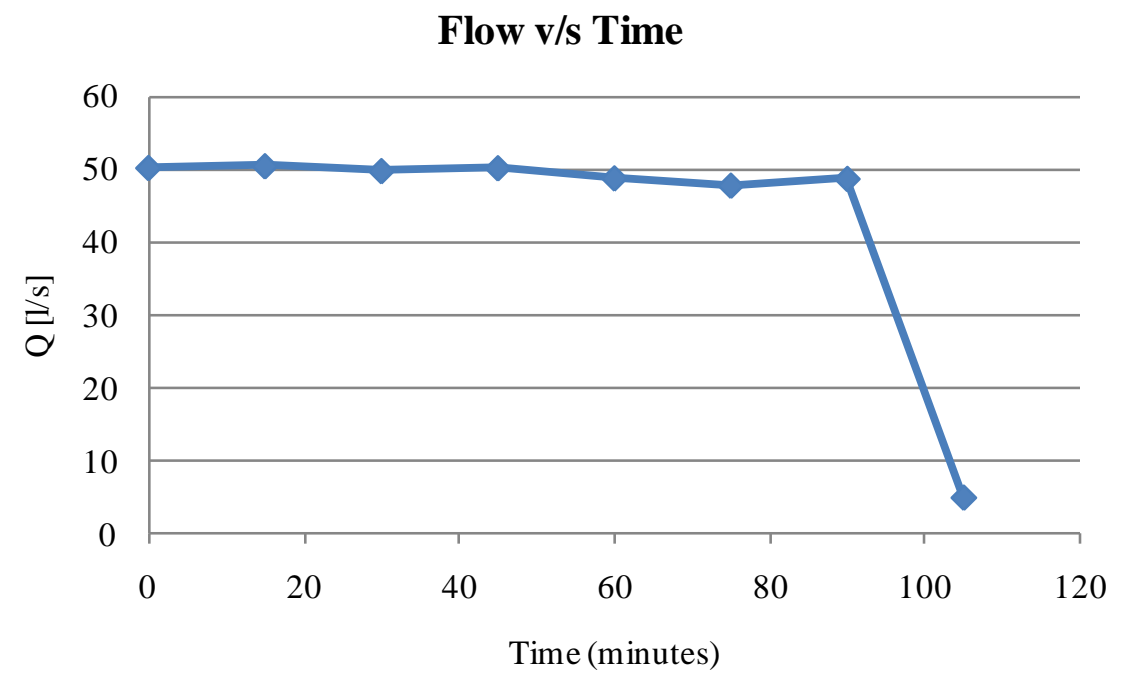

Figure 6 Variation of the flow as a function of time (Test No. 7)

The figures indicate that after approximately 1.5 hours (equivalent to a distance of $16 \mathrm{~km}$ ) in which the behaviour of the pump loop is stable, there is a sharp increase in the head loss in the pipe, which leads to a corresponding decrease of the flow rate. This indicates that there is sedimentation in the pipe as a result of a prolonged time of operation of the pump.

In the following figures, we present the variation of the friction factor and the flow during the execution of Test No. 8.

\section{Friction factor v/s Time}

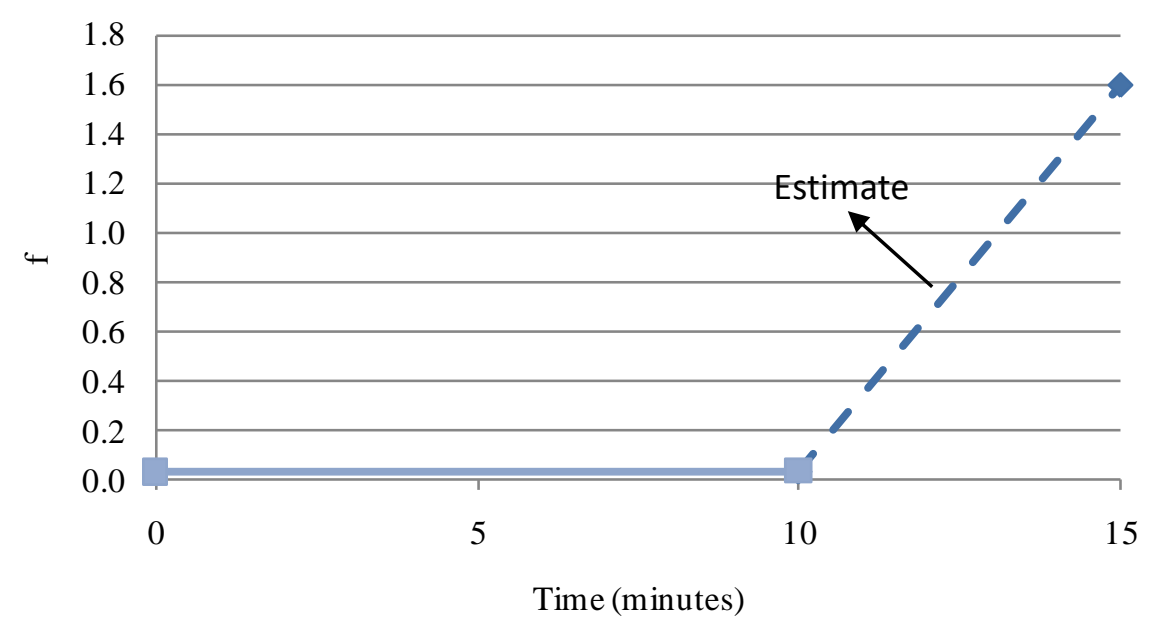

Figure 7 Variation of the friction factor measured in function of time (Test No. 8) 


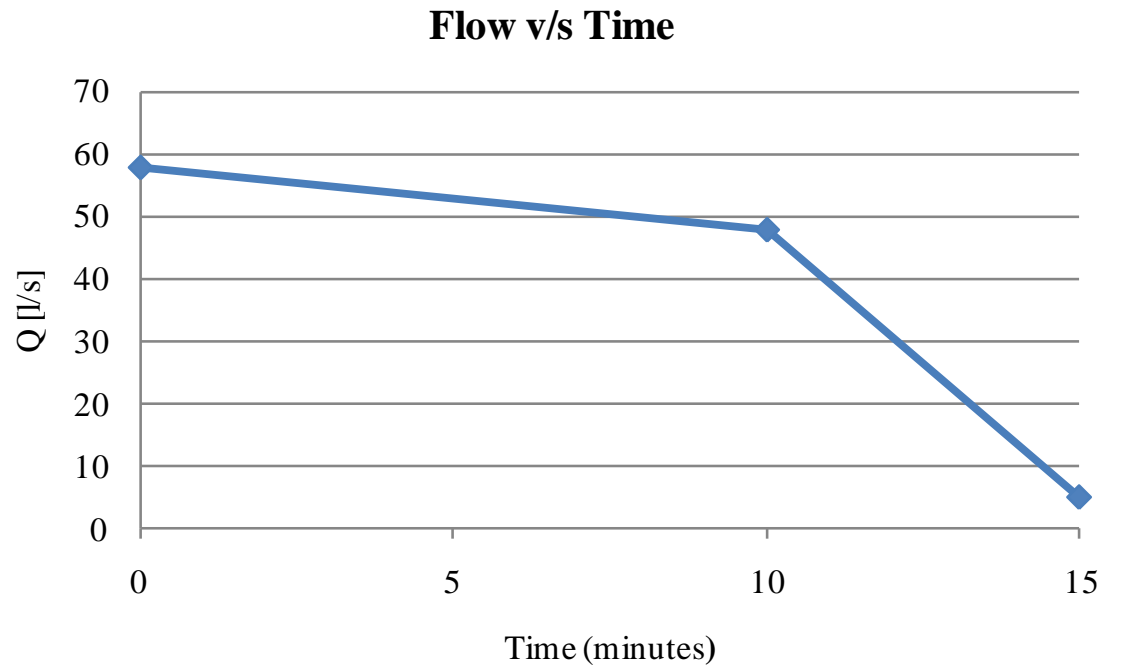

Figure 8 Variation of the flow in function of time (Test No. 8)

The figures show that after approximately 10 minutes, equivalent to a distance of $2 \mathrm{~km}$ travelled by the pulp, there is a significant increase in the friction factor and a decrease in flow that almost reaches 0 at 15 minutes of operating the loop. This indicates that in Test No. 8, sedimentation occurs in the pipe as occurred in Test No. 7, with the difference being the time this phenomenon takes.

These pump loop facilities have also been used in other tests with different tailings and no grain size distribution changes have been found at the end of the tests. Neither have relevant temperature increases during the tests occurred. Hence, particle degradation and rheology changes caused by temperature increase are not an explanation of the behaviour of long duration tests.

In both long duration tests, initially flows were relatively stable but it is possible that laminar settling out occurred and then gradually built up on the invert of the pipeline. In a closed system, a settled bed could be generated and the remaining solids keep circulating in equilibrium during pumping.

The difference between Tests 7 and 8 could be explained on the basis of the yield stress - Test 8 is more viscous than Test 7 therefore flow settles out quicker and the pressure is not enough to move the settled bed.

From an engineering perspective, these results show that it is possible using centrifugal pumps to pump tailings in laminar or laminar-turbulent transition flows during short periods of time (or short distances) and therefore, pumping short pipelines systems could be designed using these criteria.

In further investigations stationary settling out flows such as turbulent flow below settling limit velocity or laminar flow at low-pressure loss could be studied.

\section{Conclusions}

The pilot tests applied to the thickened tailings are a recognised tool to advance the knowledge of the behaviour of thickened and paste tailings, in particular when the level of concentration is close to that of paste tailings.

The measurements done for the Cerro Negro Project, in relatively simple facilities for pump loop tests, showed an expected typical tailings behaviour with laminar, turbulent and an intermediate transition zones. The results adequately adjusted to the theoretical model of Wilson and Thomas (1985) - valid for turbulent flows - and showed a wide scatter in laminar flows where the Haldenwang (2003) Model was compared with data.

The shorter tests duration do not allow us to assure if there was sedimentation inside the pipe. However, from the results of the longer tests where the pumping behaviour changed with time, we may establish 
that it is feasible that the tailings tested sediments for a solids content around $\mathrm{CW}_{\mathrm{w}} 67-68 \%$ in a transport with a significant length, for example longer than $2 \mathrm{~km}$ or even less if yield stress is extremely high.

From these tests, we have established that it is possible that tailings with behaviour predicted by the theoretical models may present sedimentation of particles in long transports, making it necessary in each case to determine the maximum solids content that will prevent the occurrence of this phenomenon. This situation limits the use of centrifugal pumps and may generate the need to use positive displacement equipment to assure the flow of the slurry at high solids concentrations and pipeline pressure gradients.

The examples presented show results useful for design and to advance in the knowledge of the behaviour of thickened tailings. For this reason, we encourage the technical community to share their experiences in this subject since it represents a light in this area where there are many factors that need to be clarified.

\section{References}

Barrera, S. and Hills, R. (2009) Cerro Negro - Assessment of Beach Slope and Indetermination of the Operational Limits of Centrifugal Pumps, in Proceedings 12th International Seminar on Paste and Thickened Tailings (Paste09), R.J. Jewell, A.B. Fourie, S. Barrera, J. Wiertz (eds), 21-24 April 2009, Viña Del Mar, Chile, Gecamin Limited, Santiago, Australian Centre for Geomechanics, Perth, pp. 337-344.

Haldenwang, R. (2003) Flow of Non-Newtonian Fluids in Open Channels, Dissertation submitted in fulfilment of the degree Doctor Technology in the Department of Civil Engineering Cape Technikon.

McElvain, R.E. and Cave, I. (1972) Transportation of Tailings in Tailing Disposal Today, in Proceedings First International Tailings Symposium. C.L. Aplin and George O. Argall (eds), 31 October-3 November, Tucson, Arizona, USA, Miller Freeman Publications, Vol.1, pp. 164-202.

Rayo, J.C. (1993) Hydraulic Transportation of Solids Over Long Distances (in Spanish), Course given for the Peruvian Mining Society, Lima, Perú.

Thomas, A.D. and Wilson, K.C. (1987) New analysis of Non-Newtonian Turbulent Flow Yield-Power-Law Fluids, The Canadian Journal of Chemical Engineering, Vol. 65, pp. 335-338.

Wilson, K.C. and Thomas, A.D. (1985) A new analysis of the turbulent flow of non-Newtonian fluids, The Canadian Journal of Chemical Engineering, Vol. 63, pp. 539-546. 